\title{
II rischio nella sperimentazione clinica dei medicinali
}

\author{
Carlo Tomino $^{1}$
}

1 Direttore Sperimentazione Clinica, Agenzia Italiana del Farmaco

La sperimentazione clinica dei farmaci, secondo le regole presenti in tutti i Paesi industrializzati, è possibile quando è sostenuta da un adeguato razionale derivato da studi in vitro e in vivo in varie specie animali che ne stabiliscano la potenziale efficacia terapeutica e l'eventuale livello di tossicità. Tuttavia, poiché l'effetto terapeutico reale può essere verificato compiutamente solo sull'uomo, la sperimentazione clinica nei pazienti diventa indispensabile per testare l'assenza di effetti dannosi, per verificare i rischi connessi all'uso del farmaco e i benefici realizzabili con il suo impiego, per conoscerne gli effetti collaterali e, infine, per metterne a punto dosaggi e modalità d'impiego.

Classicamente, nella sperimentazione clinica si identificano tre fasi: la fase I o della tollerabilità, che determina la dose massima somministrabile per un determinato periodo di tempo e il profilo di sicurezza, la fase II o dell'attività preliminare e la fase III che ha il compito fondamentale di stabilire l'efficacia e il livello rapporto rischio/beneficio per comprendere il ruolo del nuovo farmaco nella patologia indagata. A queste, normalmente, segue un'ulteriore fase (IV), successiva alla commercializzazione, che ha come scopo la conferma dell'efficacia e il monitoraggio degli effetti tossici su una popolazione più vasta.

Ogni studio clinico dovrebbe porre una domanda importante, cui si dovrebbe rispondere in modo conclusivo, tenendo sempre presente che l'obiettivo è il beneficio per il paziente.

In particolare la fase III, punto di svolta fondamentale per l'approvazione di nuovi farmaci, consiste attualmente di due studi clinici controllati e randomizzati (Randomised Controlled Trial, RCT), in cui il confronto può essere effettuato rispetto al placebo oppure a un farmaco di riferimento per l'indicazione che è oggetto dello studio.

In aggiunta a ciò, poiché la Dichiarazione di Helsinki [1] stabilisce che il placebo non può essere utiliz- zato nel caso in cui esista già un farmaco disponibile (e validato contro placebo) per una determinata indicazione, di solito i confronti si eseguono fra un nuovo farmaco e un farmaco di riferimento, utilizzato con posologia ottimale. Si deve tuttavia sottolineare che la legge europea istitutiva dell'Agenzia Europea dei Medicinali (EMEA) non richiede confronti tra medicinali ma stabilisce che un nuovo farmaco debba essere valutato sulla base di tre caratteristiche: qualità, efficacia e sicurezza [2].

Appare chiaro quindi, che la messa a punto di un nuovo farmaco è un'operazione complessa, caratterizzata da forte tecnicismo e da costi economici molto elevati. Alla ricerca e alla sperimentazione farmacologica collaborano scienziati di discipline assai diverse fra loro (chimica, biochimica, farmacologia, tossicologia, tecnica farmaceutica, biostatistica, oltre che i diversi rami della medicina), con impiego di attrezzature e procedure sofisticate.

Per i soggetti coinvolti, partecipare a una sperimentazione clinica può produrre spesso dei vantaggi, tra i quali:

- accedere alle nuove terapie che non sono ancora disponibili per tutta la popolazione;

- ottenere cure mediche presso un centro leader nell'assistenza sanitaria;

- svolgere un ruolo attivo nella cura della propria salute;

- aiutare gli altri, contribuendo al progresso della ricerca biomedica.

Ma possono esserci anche dei rischi:

- ci possono essere spiacevoli, gravi o anche pericolosi effetti collaterali prodotti dal trattamento;

- il trattamento può non essere efficace per alcuni individui;

- lo studio può richiedere molto tempo e necessitare di lunghi trattamenti, o di soggiorni in ospedale. 
Sebbene i rischi di un trial possano essere definiti, i rischi del singolo individuo o dell'intero sistema dipenderanno principalmente dal rispetto dei ruoli, dall'assunzione di responsabilità condivisa in relazione a tutto il processo e dalla capacità di controllare e gestire tutti i momenti di una sperimentazione in atto.

\section{DEFINIZIONE DEL RISCHIO NELL'AMBITO DELLA SPERIMENTAZIONE CLINICA DEI MEDICINALI}

Con il termine "pericolo" si indica qualsiasi cosa che possa provocare immediatamente danni. Il concetto di "rischio", invece, è collegato alla probabilità che un danno potenziale sia originato da un pericolo. In particolare, per quanto concerne il rischio correlato ai pazienti in trattamento all'interno di una sperimentazione clinica, si dovrà distinguere tra "rischio assoluto", ovvero la probabilità che nel soggetto coinvolto si manifestino degli eventi avversi, e "rischio relativo", cioè la probabilità che nel soggetto coinvolto si manifestino degli eventi avversi rispetto a quelli che potrebbero verificarsi con la terapia standard. Ma più in generale, nei processi di progettazione di un trial clinico vengono presi in considerazione i rischi associati allo studio, i rischi che possono provocare conseguenze, e vengono adottate tutte le misure ragionevoli per la riduzione del pericolo e delle conseguenze negative che potrebbero verificarsi. A tal fine si usa distinguere tra diverse categorie di possibili criticità in un trial, illustrate di seguito.

\section{DIRITTI}

I diritti dei pazienti arruolati comprendono:

- il consenso informato: deve essere presa in considerazione la vulnerabilità del paziente $o$ del gruppo di studio e la loro capacità di dare il consenso dopo aver ricevuto informazioni sul trial da parte di personale specificamente formato allo scopo;

- il diritto di uscire dal trial in qualsiasi momento;

- la protezione dei dati del trial e dei dati personali dei soggetti arruolati.

\section{LA SICUREZZA}

Un'altra importante tipologia di criticità in un trial concerne la sicurezza dei pazienti arruolati, e quindi i rischi a cui essi possono andare incontro. Tali rischi comprendono in primo luogo:

- le reazioni avverse al farmaco (Adverse Drug Reactions, ADR) previste e impreviste, la gestione clinica delle ADR e la gestione clinica dei pazienti. Devono pertanto essere presi in considerazione la natura degli interventi, la precedente esperienza clinica al riguardo, se il medicinale è in fase di sviluppo, le indicazioni cliniche, l'esperienza farmacologica e farmaceutica sui requisiti di gestione del farmaco in sperimentazione, la formazione e competenza del personale e infine la suscettibilità della popolazione-malattia, la genetica, l'età e il sesso;

- il probabile rapporto rischio/beneficio del trial sulla popolazione in studio: devono essere considerati i sistemi di monitoraggio e i meccanismi di valutazione delle ADR, i sistemi per mantenere la sensibilizzazione e la capacità dei partecipanti di segnalare le ADR e i risultati affidabili;

- $\quad$ i rischi dei metodi di valutazione (ad esempio biopsia, radiografia, RMN, TAC, ecc.).

Vi sono poi altri rischi di cui tener conto e che possono insorgere sia nel corso del reclutamento che nel corso del follow-up (FU); essi riguardano fattibilità, studio della popolazione, numero di soggetti necessario, competenza ed esperienza del personale, durata e frequenza del FU.

Infine va presa in esame l'affidabilità dei risultati finali, in relazione a:

- potenza dello studio;

- principali violazioni dei criteri di inclusione/ esclusione;

- procedure di randomizzazione;

- valutazione di endpoint;

- ulteriori dati: completezza e accuratezza;

- adesione al protocollo;

- potenziale malconduzione o frode.

Una volta completate le fasi di sperimentazione di un medicinale e ottenuta l'autorizzazione al commercio per una precisa indicazione terapeutica, il rapporto rischio/beneficio è giudicato positivo per la popolazione target. Tuttavia, il processo di controllo della sicurezza ed efficacia di un farmaco 
non viene interrotto, ma viene realizzato in altre forme al fine di individuare eventuali ulteriori rischi potenziali per i pazienti nella pratica clinica. Nel corso degli ultimi decenni, molte importanti problematiche di farmacovigilanza sono state identificate attraverso il meccanismo delle segnalazioni spontanee di reazioni avverse. Allo stesso tempo, si sono fatti degli sforzi per potenziare e rafforzare gli attuali sistemi di segnalazione allo scopo di fornire un nuovo tipo di approccio comune, riconosciuto dalla legislazione farmaceutica europea, in tema di gestione del rischio.

La gestione di un unico fattore di rischio è costituita da quattro fasi:

- individuazione dei rischi di base;

- valutazione del rischio;

- minimizzazione del rischio;

- comunicazione del rischio.

Tuttavia, un medicinale potrà presentare più rischi ad esso connessi e i rischi individuali potranno variare in termini di gravità, caratteristiche individuali del paziente e impatto sulla salute pubblica. Pertanto, il concetto di gestione del rischio deve riguardare anche la combinazione di informazioni su più rischi discreti con l'obiettivo di assicurare che i benefici superino i rischi, sia per il singolo paziente sia per l'intera popolazione.

Un sistema di gestione del rischio, quindi, è definito come un insieme di attività di farmacovigilanza e di interventi mirati a identificare, caratterizzare, prevenire o minimizzare i rischi connessi ai medicinali prodotti, e a valutare l'efficacia di tali interventi. Le attività per la gestione del rischio possono essere modificate da fattori come sviluppi tecnici, scientifici e legislativi come pure dalle informazioni disponibili, dai rischi percepiti e dal loro impatto sulla salute pubblica.

Quando i rischi significativi sono identificati potenzialmente già a livello degli studi pre-clinici, potrebbero essere richiesti ulteriori studi clinici per valutare il meccanismo d'azione specifico per le reazione avverse. In alcuni casi, potrebbero essere condotti ulteriori studi di farmacocinetica e farmacodinamica per determinare, per esempio, se un particolare dosaggio possa sottoporre i pazienti a un aumentato rischio di eventi avversi. Anche i test genetici potrebbero fornire indizi su quale gruppo di pazienti potrebbe essere soggetto a un aumentato rischio di reazioni avverse.

A volte, i potenziali rischi, ma anche i benefici, in popolazioni particolari potrebbero non essere pienamente quantificabili sia a causa della dimensione del campione sia a causa dell'esclusione di determinate sottopopolazioni di pazienti arruolati nelle fasi sperimentali; per esempio bambini, anziani o pazienti con condizioni co- morbose per i quali il medicinale in sperimentazione potrebbe essere metabolizzato in modo diverso rispetto al gruppo di pazienti arruolato nello studio clinico di riferimento.

In un prossimo futuro è auspicabile che le sperimentazioni cliniche possano coinvolgere sempre più anche queste popolazioni al fine di determinare esattamente l'entità del rischio e del beneficio senza l'utilizzo di modelli di trasferibilità.

\section{CONCLUSIONI}

I farmaci rappresentano ormai un bene sociale e sono parte integrante della nostra cultura; non bastano quelli che abbiamo già?

Ovviamente no, ma le risposte sono semplici e spontanee se date da un clinico, ma meno ovvie e immediate se proposte a una persona estranea a queste pratiche. Il "valore" dei farmaci nella cultura occidentale è, probabilmente, troppo spesso sottovalutato; senza ricordare alcuni dati non è facile farsi un'idea di quanto spazio occupino nella nostra vita e soprattutto di quanto migliorino il benessere e la qualità della vita. Ecco alcuni dati che è importante sottolineare (dati riferiti ai Paesi industrializzati):

- negli ultimi 30 anni l'aspettativa di vita è aumentata di 7 anni;

- la mortalità infantile è diminuita dell' $80 \%$;

- la mortalità per malattie cardiovascolari è diminuita del 50\%;

- la mortalità per le malattie dell'apparato respiratorio è diminuita del $45 \%$.

Naturalmente, a contribuire a tutto questo non è solo il farmaco, vari fattori giocano il loro ruolo, come una buona pratica clinica, uno stile di vita sano, l'aumentata igiene o una corretta alimentazione, ma non c'è dubbio che il prodotto medicinale rappresenti un importante cofattore che negli ultimi anni ha radicalmente trasformato la percezione di alcune malattie, anche quelle a decorso fatale.

Purtuttavia, non esiste il farmaco ideale, efficace al $100 \%$, privo di effetti collaterali e poco costoso. È necessario più che mai, quindi, saper agire con competenza e precisione nella valutazione delle molte variabili in gioco in tutte le fasi di progettazione e utilizzo di un nuovo farmaco, affinché i rischi siano ridotti a valori trascurabili e gli effetti siano massimizzati. Questo è uno degli scopi principali della ricerca farmacologica ed è anche uno dei suoi valori etici più alti. 


\section{BIBLIOGRAFIA}

1. World Medical Association. Declaration of Helsinki. Ethical principles for medical research involving human subjects. Helsinki: WMA General Assembly, 1964 (e successivi aggiornamenti)

2. European Parliament and the Council European Union. Regulation (EC) No 726/2004 of the European Parliament and of the Council of 31 March 2004 laying down Community procedures for the authorisation and supervision of medicinal products for human and veterinary use and establishing a European Medicines Agency. Disponibile su http://europa.eu/legislation_summaries/internal_market/single_market_for_goods/pharmaceutical_and_cosmetic_products/22149_en.htm (ultimo accesso settembre 2009) 